\title{
Medical costs and utilization in patients with depression treated with adjunctive atypical antipsychotic therapy
}

This article was published in the following Dove Press journal:

ClinicoEconomics and Outcomes Research

19 January 2013

Number of times this article has been viewed

\author{
Anagha Nadkarni' \\ Iftekhar Kalsekar' \\ Min You' \\ Robert Forbes ${ }^{2}$ \\ Tony Hebden'
}

'Bristol-Myers Squibb, Plainsboro, NJ, USA; ${ }^{2}$ Otsuka Pharmaceutical Development and Commercialization, Princeton, NJ, USA
Correspondence: Anagha Nadkarni Neuroscience Health Services, US Medical, Bristol-Myers Squibb, 777 Scudders Mill Road, Plainsboro, NJ 08536, USA

Tel +l 6098974503

Fax +I 6098976319

Email anagha.nadkarni@bms.com
Objective: To compare total medical costs and utilization over a 12 -month period in commercially insured patients receiving FDA-approved adjunctive atypical antipsychotics (aripiprazole, olanzapine, or quetiapine) for depression.

Methods: A retrospective claims analysis was conducted from 2005-2010 using the PharMetrics database. Subjects were adult commercial health-plan members with depression, identified using International Classification of Diseases codes and followed for 12 months after augmentation with an atypical antipsychotic. Outcomes included total medical costs, hospitalization, and ER visits. Generalized linear models and logistic regression were used to compare the total medical costs and the odds of hospitalization and ER visits between the treatment groups after adjusting for baseline demographic and clinical characteristics.

Results: A total of 9675 patients with depression were included in the analysis, of which $68.4 \%$ were female, with a mean age of $45.2( \pm 12.0)$ years. Adjusted 12-month total medical costs were higher for olanzapine $(\$ 14,275)$ and quetiapine $(\$ 12,998)$ compared to aripiprazole (\$9,801; $P<0.05$ for all comparisons with aripiprazole). When divided into inpatient and outpatient costs, olanzapine and quetiapine had significantly higher adjusted inpatient costs compared to aripiprazole (\$6,124 and $\$ 4,538$ vs $\$ 2,976$, respectively; $P<0.05$ for all comparisons with aripiprazole). Similar results were seen for adjusted outpatient costs. Adjusted odds of hospitalization for olanzapine (odds ratio $[\mathrm{OR}]=1.73 ; 95 \% \mathrm{CI}$ confidence interval $[\mathrm{CI}]=1.42-2.10)$ and quetiapine $(\mathrm{OR}=1.40 ; 95 \% \mathrm{CI}=1.21-1.60)$ were significantly higher than aripiprazole at 12 months. The adjusted odds of an ER visit for olanzapine (OR = 1.40; $95 \% \mathrm{CI}=1.18-1.65)$ and quetiapine $(\mathrm{OR}=1.62 ; 95 \% \mathrm{CI}=1.44-1.81)$ were also significantly higher compared to aripiprazole at 12 months.

Conclusions: In commercially insured major depressive disorder patients, olanzapine and quetiapine were associated with higher total medical costs, the difference being primarily attributable to higher inpatient costs. Additionally, olanzapine and quetiapine were associated with significantly higher odds of hospitalization and ER visits compared to aripiprazole.

Keywords: depression, atypical antipsychotics, adjunctive therapy

\section{Background}

Major depressive disorder (MDD) is a prevalent, disabling mental health disorder that affects an estimated 14 million adults in the US each year. ${ }^{1}$ The lifetime prevalence of MDD ranges from $16 \%$ to $18 \%$, translating to over 35 million adults in the US being affected at some point during their life. ${ }^{1,2}$ The economic burden associated with MDD is also substantial, with a total cost burden estimate of $\$ 83$ billion in 2000 (of which $\$ 26.1$ billion was attributable to direct medical costs). ${ }^{3}$ More recent estimates determined the 2-year total cost burden in 2007 dollars to be $\$ 20,976$ for 
patients responding to therapy, and $\$ 32,537$ for patients not responding adequately to therapy. ${ }^{4}$

Despite the numerous agents available for the treatment of depression, patients who receive treatment do not always respond adequately to antidepressant therapy (ADT) ${ }^{5,6}$ For example, less than half $(48.6 \%)$ of patients responded to first-line treatment in the Sequenced Treatment Alternatives to Relieve Depression (STAR*D) trial..$^{5}$ Other research has demonstrated that only $50 \%-60 \%$ of patients respond to first-line treatment, indicating that a significant portion of patients with depression have an inadequate response to ADT and require further treatment. ${ }^{7}$ Not only does this inadequate response to ADT present a clinical complication, it also has economic implications. ${ }^{8}{ }^{8}$ Studies have shown that patients who respond inadequately to ADT experience a disproportionate burden of illness, ${ }^{4,9,10}$ particularly as the disease increases in severity. ${ }^{11}$ Direct medical costs have been shown to be $40 \%$ higher among inadequate responders compared to patients who respond to therapy. ${ }^{12}$

A range of augmentation and combination strategies are used by physicians to improve outcomes for patients with depression who exhibit an inadequate response to ADT. The American Psychiatric Association guidelines recommend a change in dose, switching to a new drug, or adjunctive or augmentation therapy in patients who either do not respond to treatment or achieve only a partial response to therapy during an acute phase..$^{13}$ One option for adjunctive therapy in patients with an inadequate response is augmenting ADT with an atypical antipsychotic. Currently, aripiprazole, olanzapine, and quetiapine are the only atypical antipsychotics indicated for adjunctive therapy in depression.

Though the clinical benefits and efficacy of these agents as adjunctive treatment is well documented, ${ }^{14,15}$ there are limited data on the comparative effectiveness of atypical antipsychotics in the real-world setting. ${ }^{6}$ To date, only one study evaluating the real-world comparative effectiveness of the three indicated atypical antipsychotics has been published. ${ }^{16}$ Using a commercial population, Jing et al determined that over a 6-month period, patients treated with olanzapine or quetiapine had higher total health-care and medical costs compared to patients receiving aripiprazole. Adjusted risk for hospitalizations and ER visits were found to be significantly higher for quetiapine compared to aripiprazole. ${ }^{16}$

The current study adds to the literature by using data from a different commercially insured population, as well as by examining outcomes over a longer follow-up period in order to capture the long-term effects of treatment with adjunctive atypical antipsychotics. Furthermore, while the
Jing et al study included only those patients who had newly initiated atypical antipsychotics as their first augmentation option, this study included all patients newly initiating atypical antipsychotics, irrespective of whether or not the atypical antipsychotics were initiated as the first-line augmentation strategy. The objective of the current study was to compare the total medical costs and utilization in patients receiving an indicated atypical antipsychotic as adjunctive therapy for depression.

\section{Methods Data source}

This was a retrospective cohort study that utilized PharMetrics data from 2005 to 2010. The PharMetrics database contains de-identified commercial insurance claims of more than 70 million members from more than 100 health plans across the US. The database includes inpatient and outpatient medical claims, diagnosis and procedure codes, and pharmacy claims. The PharMetrics database is geographically representative of the US population and includes a variety of demographic measures. ${ }^{17}$

\section{Patient selection}

Patients who were 18-64 years of age, with a diagnosis of depression based on an International Classification of Diseases, Ninth Revision, Clinical Modifications (ICD-9 CM) diagnosis code (296.2x, 296.3x, 311.xx) were selected (Figure 1). The cohort was also restricted to those patients who filled a prescription for aripiprazole, olanzapine, or quetiapine, defined as the index atypical antipsychotic, from July 1, 2005 to July 1, 2009. The date on which the patient first filled a prescription for one of these three agents was set as the index date. Patients were required to have 6 months continuous enrollment in the preindex period and 12 months continuous enrollment in the postindex period, resulting in a study period of January 1, 2005 to June 30, 2010. A minimum of 60 days supply of ADT during the preindex period was necessary, as was a minimum of 14 overlapping days supply between the index atypical antipsychotic agent and the first ADT prescription. To ensure concurrent use, this 14-day overlap was required to occur within the first 90 days postindex date.

Patients with an ICD-9 CM code for bipolar disorder (296.0x, 296.1x, 296.4x, 296.5x, 296.6x, 296.80, 296.81, 296.89) or schizophrenia (295.xx) at any point during the study period were excluded. Patients were also excluded if they had had a fill for an atypical antipsychotic anytime during the 6-month preindex period. 


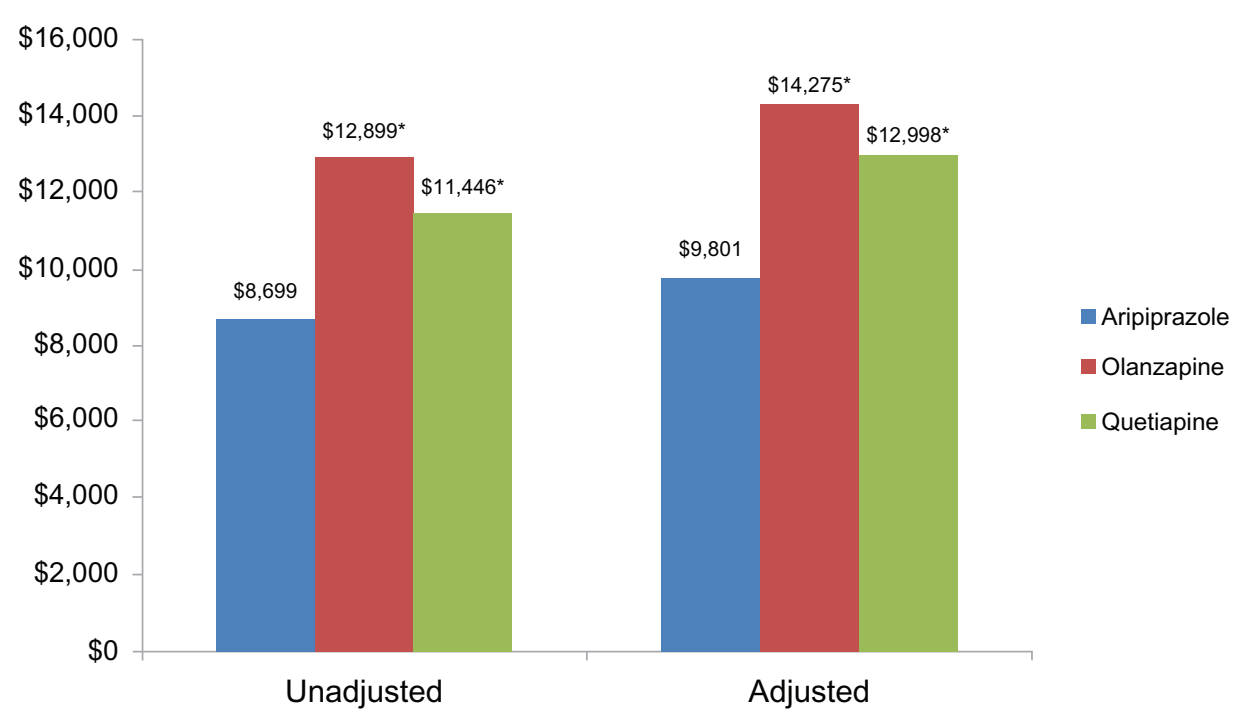

Figure I Twelve-month unadjusted and adjusted ${ }^{\mathrm{a}}$ total medical costs.

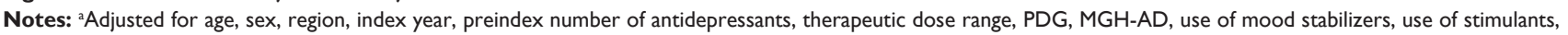
use of thyroid, visit to a psychiatric provider, MDD code of $296.2 x$ or $296.3 x$, diabetes, hyperlipidemia. $* P<0.05$ for all comparisons with aripiprazole.

Abbreviations: MDD, major depressive disorder; MGH-AD, Massachusetts General Hospital Scale - antidepressant version; PDG, psychiatric diagrosis group.

Patient selection ultimately resulted in three mutually exclusive cohorts based on the index atypical antipsychotic.

\section{Outcomes}

Patients were followed for 12 months, and outcomes were measured over the 12-month postindex period using an intent-to-treat methodology. Total medical costs were evaluated for the 12-month period, and were further stratified into inpatient (hospitalizations and ER visits) and outpatient costs (physician office visits and labs). Total medical costs included all costs, not just those pertaining to depression. Pharmacy costs were not included in the analysis. Current relative pharmacy costs likely differ from pharmacy costs when this study was conducted, due to the recent introduction of generic olanzapine and quetiapine; therefore, for this analysis we focused on the relative total medical costs of patients using each medication, which will likely remain consistent between the study period and the present day. Costs were computed based on health-plan paid amounts for each claim. All cost measures were adjusted to 2010 dollars using the annual medical-care component of the Consumer Price Index to account for inflation between 2005 and 2010. Outcomes also included occurrence of hospitalization and ER visits during the 1-year follow-up period.

\section{Covariates}

The following covariates, being demographic characteristics, comorbid conditions, and proxies for disease severity, were measured.

\section{Demographic characteristics}

Patient demographic characteristics included age, sex, region (US region in which the patient was enrolled, categorized as East, West, Midwest, and South), and the year of the index date.

\section{Comorbid conditions}

The Deyo version of the Charlson Comorbidity Index (CCI) score and the mean number of psychiatric diagnosis groups (PDGs) were computed as a measure of comorbidity. ${ }^{18-20}$ The CCI estimates the burden of comorbid conditions and is a numerical scale based on the presence or absence of 19 conditions/disease states. Each condition is assigned a weight, with higher scores indicating increased burden. PDGs include conditions such as mental disorders that are not included as part of the CCI. Counts for PDGs were calculated, with a range from 0 to 12 . In addition, the presence of diabetes or hyperlipidemia was noted as a dichotomous variable. Since atypical antipsychotics differ in their metabolic side-effect profile, there could be a channeling bias for use of specific atypical antipsychotics based on the presence of these conditions at baseline, thus resulting in the need to control for these specific covariates.

\section{Disease severity}

The Massachusetts General Hospital scale - antidepressant version (MGH-AD) was incorporated as a covariate to measure the level of treatment resistance. The score is calculated by assigning 1 point for each adequate ADT trial, and 0.5 points for each optimization strategy (extended duration 
or titration). Higher scores indicate more severe disease, and a score of greater than 3 is considered indicative of treatment resistance. ${ }^{12,20}$ In addition, the number of ADTs in the preindex period was tabulated and categorized as 1, 2, or 3+ADTs. Therapeutic dose range for atypical antipsychotics was also calculated based on the FDA label dosing recommendations. Therapeutic ranges were defined as $5-15 \mathrm{mg}$ for aripiprazole, 6-20 mg for olanzapine, and 150-300 mg for quetiapine..$^{21-23}$ Additional disease-severity covariates captured included the use of mood stabilizers, stimulants, thyroid medications, visit to a specialist provider (psychiatrist), and diagnosis of depression based on ICD-9 codes of $296.2 x$ or $296.3 x$ versus identification based solely on the ICD-9 code of 311 .xx. The hospitalization and ER-visit analyses included a dichotomous covariate to control for baseline hospitalization/ER visits.

All covariates were measured on the index date or in the 6-month preindex period, with the exception of therapeutic dose range.

\section{Statistical analysis}

Univariate comparisons of baseline characteristics and outcome variables between the olanzapine or quetiapine cohorts to the aripiprazole cohort were conducted using $t$-test and Chi-square tests (for continuous and categorical variables, respectively).

Adjusted total medical costs were estimated using the generalized linear model with gamma distribution and log-link transformation to account for the abnormal distribution of costs. Total medical costs were split into inpatient and outpatient costs. For the analysis of inpatient costs (cost of hospitalization and ER visits), a two-stage multivariate modeling approach was used, combining logistic regression, generalized linear model, and bootstrapping with 200 repetitions, all to account for the fact that many beneficiaries had no hospitalizations/ER visits and therefore incurred no inpatient/ER costs. Adjusted odds of total hospitalization and adjusted odds of total ER visits were also estimated using logistic regression. Odds ratios (ORs) were calculated for all hospitalizations and ER visits, and were not mental health-specific. All multivariate models controlled for demographics, comorbid conditions, and disease-severity proxies listed in the Covariates section.

Adjusted mean total medical costs and utilization rates were calculated at a population level by taking the mean values of the covariates for all patients in the study.

The base-case analysis for the study used an intentto-treat approach with outcomes being measured over a 12-month period. However, in this study the atypical antipsychotics had an average duration of therapy ranging from 4 to 5 months. Given this average duration of therapy, sensitivity analyses were conducted to examine outcomes at 6 months, in order to test the robustness of the results from the base-case analysis. Additionally, sensitivity analyses were also conducted for utilization outcomes using Cox proportional hazard $(\mathrm{CPH})$ models to evaluate the time-dependent risk of hospitalization and ER visits after controlling for demographic characteristics, comorbid conditions, and disease-severity proxies. Patients were followed from index until one of the following events occurred: (1) date of discontinuation (run-out date of the last prescription filled prior to a 30-day gap in therapy), (2) date of switch to another atypical antipsychotic, (3) end of the follow-up period (6 months or 12 months), or (4) occurrence of outcome (hospitalization or ER visit).

All analyses were conducted using SAS version 9.1 (SAS Institute, Cary, NC) with an a priori significance level of 0.05 .

\section{Results}

\section{Patient sample and baseline characteristics}

Demographic and baseline characteristics are presented in Table 1. The sample was comprised of 9675 patients with depression: 3142 (32.5\%) in the aripiprazole cohort, $945(9.8 \%)$ in the olanzapine cohort, and 5588 (57.7\%) in the quetiapine cohort. Overall, there were more females (68.4\%) than males, and the average age of the sample was $45.2( \pm 12.0)$ years. More than half of patients $(55.3 \%)$ had more than one unique ADT in the preindex period.

The mean age of patients in the aripiprazole cohort was significantly lower than patients in the olanzapine cohort $(P<0.001)$. Additionally, the aripiprazole cohort had significantly more females compared to the olanzapine and quetiapine cohorts $(P<0.001$ for all). The distribution of patients varied by geographic region across the cohorts $(P<0.01$ for all), but despite differences in overall geographic locations, the majority of patients in each cohort were from the East or Midwest regions.

The aripiprazole cohort had a significantly lower comorbid burden as measured by the Deyo CCI score compared to olanzapine and quetiapine, as well as a significantly lower PDG score compared to the quetiapine cohort.

For disease severity, the aripiprazole cohort had a significantly higher MGH-AD score (2.2 vs 2.0 for other cohorts; $P<0.001$ for each comparison). Furthermore, a significantly higher percentage of patients in the 
Table I Characteristics of patients treated with adjunctive aripiprazole, olanzapine, or quetiapine

\begin{tabular}{|c|c|c|c|c|c|}
\hline & $\begin{array}{l}\text { Aripiprazole } \\
(n=3142)\end{array}$ & $\begin{array}{l}\text { Olanzapine } \\
(n=945)\end{array}$ & $P$-value & $\begin{array}{l}\text { Quetiapine } \\
(n=5588)\end{array}$ & $P$-value \\
\hline \multicolumn{6}{|l|}{ Demographics } \\
\hline Age, mean (SD) & $45.1(11.9)$ & 46.9 (II.7) & $<0.001$ & $44.8(I 1.8)$ & 0.220 \\
\hline \multicolumn{6}{|l|}{ Sex, \% (n) } \\
\hline Women & $72.4(2,276)$ & $62.1(587)$ & $<0.001$ & $68.1(3803)$ & 0.0001 \\
\hline \multicolumn{6}{|l|}{ Region, \% (n) } \\
\hline East & $30.5(957)$ & $29.1(275)$ & & $29.4(1644)$ & \\
\hline Midwest & $42.4(1333)$ & 42.1 (398) & & $43.3(2419)$ & \\
\hline West & $5.9(186)$ & $9.0(85)$ & & $7.9(443)$ & \\
\hline South & $21.2(666)$ & $19.8(187)$ & $<0.01$ & $19.4(1082)$ & $<0.01$ \\
\hline \multicolumn{6}{|l|}{ Index year, \% (n) } \\
\hline 2005 & $3.8(119)$ & $10.5(99)$ & & $8.5(474)$ & \\
\hline 2006 & $9.9(3 \mid I)$ & $24.3(230)$ & & $22.2(1238)$ & \\
\hline 2007 & $14.3(450)$ & $30.3(286)$ & & $30.8(1723)$ & \\
\hline 2008 & $46.6(1463)$ & $27.8(263)$ & & $30.9(1726)$ & \\
\hline 2009 & 25.4 (799) & $7.1(67)$ & $<0.001$ & $7.6(427)$ & $<0.001$ \\
\hline \multicolumn{6}{|l|}{ Comorbid conditions } \\
\hline Deyo CCl, mean (SD) & $0.4(1.0)$ & $0.6(1.3)$ & $<0.00$ I & $0.5(1.2)$ & $<0.001$ \\
\hline PDG, mean (SD) & $1.5(1.1)$ & $1.5(1.3)$ & 0.873 & $1.8(1.3)$ & $<0.001$ \\
\hline Diabetes, \% (n) & $6.9(218)$ & $5.8(55)$ & 0.227 & $6.6(366)$ & 0.486 \\
\hline Hyperlipidemia, \% (n) & $14.5(456)$ & $14.2(134)$ & 0.798 & I4.I (788) & 0.598 \\
\hline \multicolumn{6}{|l|}{ Disease-severity proxies } \\
\hline \multicolumn{6}{|l|}{ Preindex number of antidepressants, \% (n) } \\
\hline I & $46.8(|47|)$ & $45.8(433)$ & & $43.3(2422)$ & \\
\hline 2 & $37.9(1190)$ & $36.5(345)$ & & $38.4(2 \mid 44)$ & \\
\hline $3+$ & $15.3(48 \mathrm{I})$ & $17.7(167)$ & 0.215 & $18.3(1022)$ & $<0.001$ \\
\hline Dose within therapeutic range at 6 months, \% (n) & $68.1(2139)$ & $41.3(390)$ & $<0.001$ & $22.8(1273)$ & $<0.001$ \\
\hline Dose within therapeutic range at 12 months, $\%$ (n) & $68.9(2165)$ & $42.9(405)$ & $<0.001$ & $24.1(1347)$ & $<0.001$ \\
\hline MGH-AD, mean (SD) & $2.2(1.1)$ & $2.0(1.2)$ & $<0.001$ & $2.0(1.2)$ & $<0.001$ \\
\hline Preindex mood stabilizer, \% (n) & $30.8(969)$ & $26.1(247)$ & $<0.01$ & $28.1(|57|)$ & $<0.01$ \\
\hline Preindex stimulant, \% (n) & 14.4 (454) & $7.0(66)$ & $<0.001$ & $8.2(456)$ & $<0.001$ \\
\hline Preindex thyroid, \% (n) & $13.9(436)$ & $12.0(1 \mid 3)$ & 0.129 & $10.8(606)$ & $<0.001$ \\
\hline Visit to psychiatric provider, \% (n) & $66.4(2086)$ & $54.0(510)$ & $<0.001$ & $63.5(3548)$ & $<0.01$ \\
\hline Diagnosis of MDD based on ICD-9 codes & $78.6(2469)$ & $67.0(633)$ & $<0.001$ & $74.0(4 \mid 35)$ & $<0.001$ \\
\hline \multicolumn{6}{|l|}{ of $296.2 x$ or $296.3 x, \%(n)$} \\
\hline Hospitalization, \% (n) & I5.I (475) & $23.3(220)$ & $<0.001$ & $27.7(1548)$ & $<0.001$ \\
\hline ER visit, \% (n) & $25.3(795)$ & $33.3(3 \mid 5)$ & $<0.001$ & $39.3(2197)$ & $<0.001$ \\
\hline
\end{tabular}

Abbreviations: SD, standard deviation; CCl, Charlson Comorbidity Index; PDG, psychiatric diagnosis group; MGH-AD, Massachusetts General Hospital scale - antidepressant version; MDD, major depressive disorder; ICD, International Classification of Diseases; ER, Emergency room.

aripiprazole cohort (78.6\%) had a definitive MDD diagnosis based on ICD-9 codes of 296.2x (MDD single episode) or 296.3x (MDD recurrent episode) as opposed to a diagnosis based on a broader ICD-9 code of 311.xx (depressive disorder, not elsewhere classified). Significantly more patients in the aripiprazole cohort had used mood stabilizers ( $30.8 \%$ vs $26.1 \%$ and $28.1 \%$ for olanzapine and quetiapine, respectively; $P<0.01$ for all comparisons with aripiprazole), and stimulants ( $14.4 \%$ vs $7.0 \%$ and $8.2 \%$ for olanzapine and quetiapine, respectively; $P<0.001$ for all comparisons with aripiprazole). Furthermore, more aripiprazole-treated patients had visited a psychiatrist ( $66.4 \%$ vs $54.0 \%$ and $63.5 \%$ for olanzapine and quetiapine, respectively; $P<0.01$ for all comparisons with aripiprazole). Compared to patients in the quetiapine cohort, significantly more patients in the aripiprazole cohort used thyroid medications $(13.9 \%$ vs $10.8 \%$, $P<0.001)$. In addition, a significantly higher proportion of patients in the aripiprazole cohort were dosed within the indicated therapeutic dosing range at 6 months (68.1\%) compared to patients in the olanzapine or quetiapine cohorts (41.3\% and $22.8 \%$, respectively; $P<0.001$ for all comparisons with aripiprazole).

\section{Costs}

Unadjusted 12-month total medical costs were significantly higher for olanzapine $(\$ 12,899)$ and quetiapine 
$(\$ 11,446)$ compared to aripiprazole $(\$ 8,669 ; P<0.05$ for all comparisons with aripiprazole) (Figure 2). When costs were adjusted for patient demographics, comorbidities, and disease severity, the trends and significance were maintained. Adjusted total medical costs for patients on olanzapine $(\$ 14,275)$ and quetiapine $(\$ 12,998)$ were significantly higher than patients on aripiprazole $(\$ 9,801 ; P<0.05$ for all comparisons with aripiprazole) (Figure 1).

When total medical costs were divided into outpatient and inpatient costs, both were significantly higher for patients on olanzapine ( $\$ 6,268$ and $\$ 6,124$; respectively) and quetiapine (\$6,874 and $\$ 4,538$; respectively) compared to aripiprazole (\$5,679 and $\$ 2,976$; respectively; $P<0.05$ for all comparisons with aripiprazole) (Figure 2).

\section{Hospitalization and ER visits}

Unadjusted 12-month hospitalization rates were significantly higher for olanzapine $(24.6 \%, P<0.001)$ and quetiapine (22.0\%, $P<0.001)$ compared to the aripiprazole cohort (15.4\%). Similarly, unadjusted 12-month ER visits for olanzapine and quetiapine were significantly higher relative to the aripiprazole cohort (36.5\% and $41.4 \%$ vs $29.7 \%$, respectively; $P<0.001$ for all comparisons) (Table 2 ).

Adjusted odds of hospitalization for olanzapine $(\mathrm{OR}=1.73 ; 95 \%$ confidence interval $[\mathrm{CI}]=1.42-2.10)$ and quetiapine $(\mathrm{OR}=1.40 ; 95 \% \mathrm{CI}=1.21-1.60)$ were significantly higher compared to aripiprazole at 12 months. The odds of ER visits for olanzapine $(\mathrm{OR}=1.40$; $95 \% \mathrm{CI}=1.18-1.65)$ and quetiapine $(\mathrm{OR}=1.62 ; 95 \%$
$\mathrm{CI}=1.44-1.81)$ were also significantly higher compared to aripiprazole at 12 months (Figure 3). Adjusted rates of hospitalization were significantly higher for olanzapine and quetiapine compared to aripiprazole $(30.3 \%$ and $26.0 \%$ vs $20.1 \%$, respectively; $P<0.001$ for all comparisons with aripiprazole). ER visits for olanzapine and quetiapine were also significantly higher than aripiprazole $(29.6 \%$ and $32.8 \%$ vs $23.1 \%$, respectively; $P<0.001$ for all comparisons with aripiprazole) (Table 2).

\section{Sensitivity analysis}

Results at 6 months were consistent with results from the base-case analysis for both costs and utilization. The olanzapine and quetiapine cohorts had significantly higher unadjusted and adjusted total medical costs at 6 months (adjusted total medical costs at 6 months were $\$ 7,613$ for olanzapine and $\$ 6,552$ for quetiapine vs $\$ 4,876$ for aripiprazole; $P<0.05$ for all comparisons with aripiprazole). Unadjusted and adjusted rates as well as ORs for hospitalization and ER visits for olanzapine and quetiapine at 6 months were significantly higher compared to aripiprazole (data not shown).

Both olanzapine (hazard ratio $[\mathrm{HR}]=2.10 ; 95 \%$ $\mathrm{CI}=1.62-2.72)$ and quetiapine $(\mathrm{HR}=1.82 ; 95 \% \mathrm{CI}=1.50-2.20)$ had significantly higher hazard of hospitalization at 6 months compared to aripiprazole based on CPH models. In addition, olanzapine $(\mathrm{HR}=1.41 ; 95 \% \mathrm{CI}=1.16-1.70)$ and quetiapine $(\mathrm{HR}=1.42 ; 95 \% \mathrm{CI}=1.24-1.61)$ had a significantly higher hazard of ER visits at 6 months compared to aripiprazole. Olanzapine and quetiapine also had a significantly higher

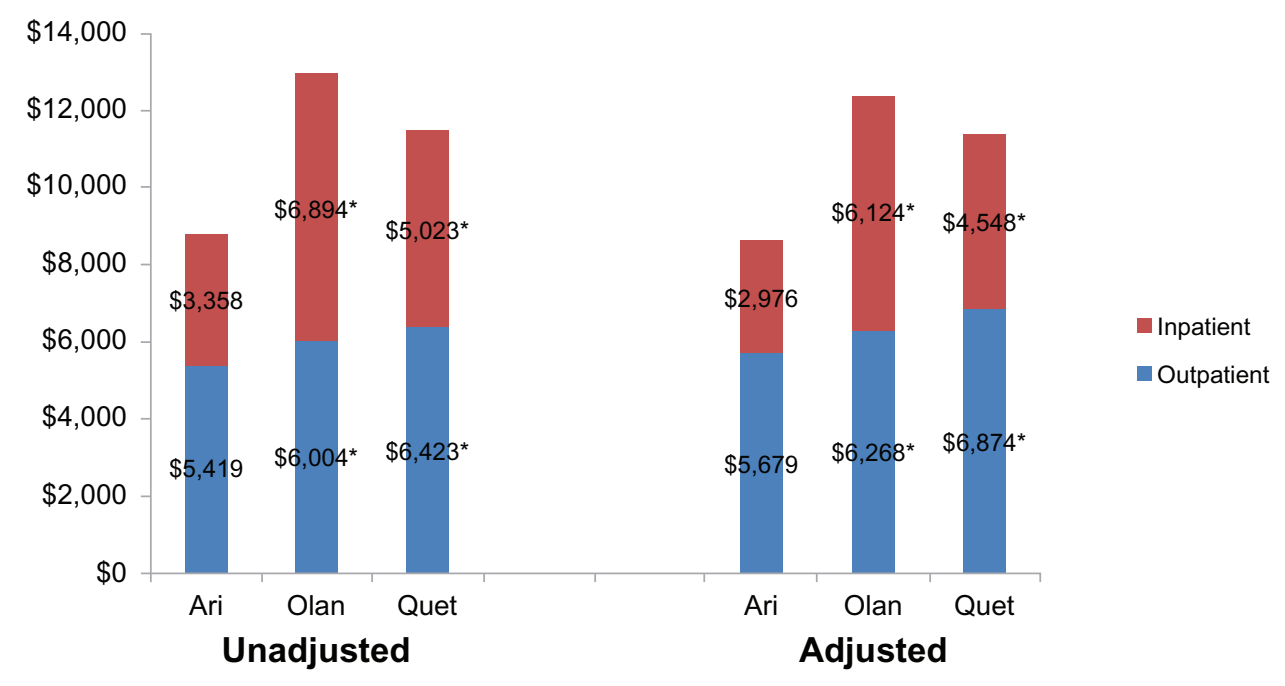

Figure 2 Twelve-month unadjusted and adjusted ${ }^{\mathrm{a}}$ inpatient and outpatient costs.

Notes: ${ }^{a}$ Adjusted for age, sex, region, index year, pre-index number of antidepressants, therapeutic dose range, PDG, MGH-AD, use of mood stabilizers, use of stimulants, use of thyroid, visit to a psychiatric provider, MDD code of $296.2 x$ or $296.3 x$, diabetes, hyperlipidemia. $* P<0.05$ for all comparisons with aripiprazole.

Abbreviations: Ari, aripiprazole; Olan, olanzapine; Quet, quetiapine; MDD, major depressive disorder; MGH-AD, Massachusetts General Hospital Scale - antidepressant version; PDG, psychiatric diagnosis group. 
Table 2 Unadjusted and adjusted ${ }^{a}$ 12-month postindex rates for hospitalization and ER visits

\begin{tabular}{|c|c|c|c|}
\hline & $\begin{array}{l}\text { Aripiprazole } \\
(n=3142)\end{array}$ & $\begin{array}{l}\text { Olanzapine } \\
(n=945)\end{array}$ & $\begin{array}{l}\text { Quetiapine } \\
(n=5588)\end{array}$ \\
\hline \multicolumn{4}{|c|}{ Unadjusted I2-month postindex rates of hospitalization and ER visits } \\
\hline Hospitalization, \% (n) & $15.4(485)$ & $24.6(232)^{b}$ & $22.0(1227)^{\mathrm{b}}$ \\
\hline ER visits, \% (n) & $29.7(932)$ & $36.5(345)^{b}$ & $4 I .4(2,3 \mid 5)^{b}$ \\
\hline \multicolumn{4}{|c|}{ Adjusted $^{\mathrm{a}}$ I2-month postindex rates of hospitalization and ER visits } \\
\hline Hospitalization & $20.1 \%$ b & $30.3 \%$ b & $26.0 \%$ b \\
\hline ER visits & $23.1 \%$ & $29.6 \%$ & $32.8 \%{ }^{\mathrm{b}}$ \\
\hline
\end{tabular}

Notes: ${ }^{a} A d j u s t e d$ for age, sex, region, index year, pre-index number of antidepressants, therapeutic dose range, PDG, MGH-AD, use of mood stabilizers, use of stimulants, use of thyroid, visit to a psychiatric provider, MDD code of $296.2 x$ or $296.3 x$, diabetes, hyperlipidemia, preindex hospitalization or ER visit; ${ }^{b} P<0.00$ I for all comparisons to aripiprazole.

Abbreviations: $E R$, Emergency room; MDD, major depressive disorder; MGH-AD, Massachusetts General Hospital Scale - antidepressant version; PDG, psychiatric diagnosis group.

hazard of hospitalization and ER visits at 12 months compared to aripiprazole based on $\mathrm{CPH}$ models.

\section{Discussion}

This study compared total medical costs and utilization among patients with depression who received adjunctive therapy with one of three indicated atypical antipsychotics: aripiprazole, olanzapine, or quetiapine. Based on the results of this study, patients who were treated with aripiprazole had significantly lower total medical costs compared to patients treated with olanzapine or quetiapine. To further understand the differences across the cohorts, total medical costs were divided into inpatient and outpatient costs. Results showed that inpatient costs were a significant component of the total medical costs for olanzapine and quetiapine cohorts and were significantly higher compared to inpatient costs for patients

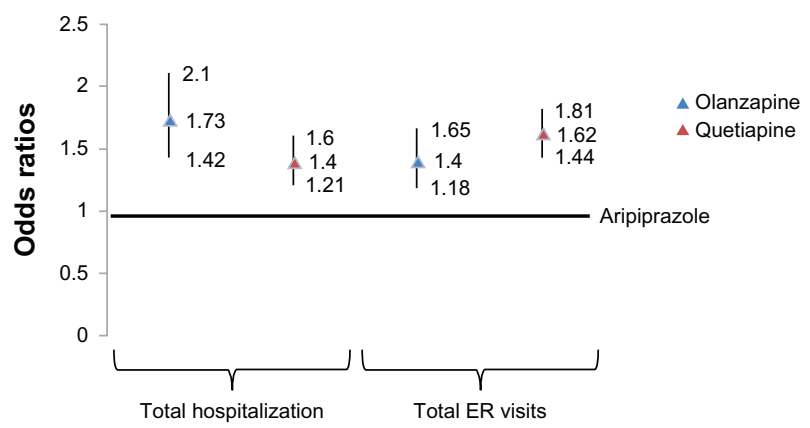

Figure 3 Adjusted $^{\text {a }} 12$ month post-index odds of hospitalization ER visits compared to aripiprazole.

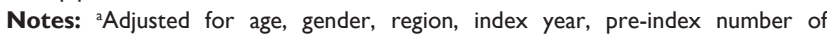
antidepressants, therapeutic dose range, PDG, MGH-AD, use of mood stabilizers, use of stimulants, use of thyroid, visit to a psych provider, MDD code of $296.2 x$ or 296.3x, diabetes, hyperlipidemia, pre-index hospitalization or ER visit.

Abbreviations: ER, Emergency room; MDD, major depressive disorder; $M G H-A D$, Massachusetts General Hospital Scale - antidepressant version; PDG, psychiatric diagnosis group. on aripiprazole. Consistent with this, it was found that the aripiprazole cohort had significantly lower hospitalizations and ER visits compared to both olanzapine and quetiapine.

Recently, Jing et al carried out a study to evaluate healthcare costs and utilization in patients with depression receiving atypical antipsychotics as adjunctive therapy. ${ }^{16}$ While the two studies would appear similar, the current study had a longer follow-up period (12 months vs 6 months), was conducted in a different commercial population and used more recent data. Furthermore, while the Jing et al study focused solely on patients who were newly initiated with atypical antipsychotics as a first-line augmentation strategy, the current study examined all patients newly initiated with atypical antipsychotics, irrespective of whether the atypical antipsychotic was a first-line augmentation strategy. Despite these differences, the results were consistent, demonstrating that total medical costs were significantly lower for aripiprazole-treated patients than olanzapine- and quetiapine-treated patients. In the study by Jing et al, adjusted 6-month total medical costs (in 2009 dollars) were $\$ 6,062$ for olanzapine and $\$ 7,298$ for quetiapine, compared to $\$ 3,986$ for aripiprazole ( $P=0.02$ and $P<0.01$ respectively). The current study also revealed that 12 -month total medical costs (in 2010 dollars), were significantly higher for patients in the olanzapine $(\$ 14,275)$, and quetiapine $(\$ 12,998)$ cohorts compared to patients in the aripiprazole cohort $(\$ 9,801$; $P<0.05$ for all comparisons with aripiprazole). ${ }^{16}$ While the results were consistent between the two studies, the Jing et al study had slightly lower monthly total medical costs, which may be due to the fact that the patients in that study had less disease severity than patients in the current study. This discrepancy in disease severity across the two studies is likely because the current study focused on all patients newly initiated with atypical antipsychotics regardless of line of therapy, resulting in a population with greater disease severity compared to the Jing et al population. Results for utilization outcomes were also consistent, as in both studies the adjusted relative risk of hospitalization for quetiapine-treated patients was significantly higher compared to aripiprazole-treated patients. The adjusted risk for hospitalization was numerically higher for the olanzapine cohort compared to the aripiprazole cohort in the Jing et al study, but it did not reach statistical significance. Consistent with this, Jing et al demonstrated that ER visits mirrored total hospitalization results, with significant differences for the quetiapine cohort and numerically higher values for the olanzapine cohort that did not reach significance compared to the aripiprazole cohort. ${ }^{16}$ These differences may be attributable to differences in patient populations or 
differences in follow-up time. Overall, however, the trends between the studies were consistent.

Other researchers have attempted to quantify and compare the effectiveness of atypical antipsychotics in MDD. A decision analytic model was constructed by Taneja et al to evaluate the cost-effectiveness of indicated atypical antipsychotics in patients with MDD. ${ }^{24}$ The model inputs were based on clinical trial data and were used to calculate the cost per responder. Patients receiving aripiprazole had the lowest cost per additional responder $(\$ 3,447)$, followed by olanzapine/fluoxetine $(\$ 3,993)$, quetiapine $300 \mathrm{mg} /$ day $(\$ 6,000)$, and quetiapine $150 \mathrm{mg} /$ day $(\$ 8,725) .{ }^{24}$ It should be noted that the results were based on inputs from clinical trial data, and on specific FDA-approved dosing ranges for each agent, so are less reflective of the real world than the current study.

The reason for the differences observed in the current study is not known. It was found that real-world dosing differed among the patient populations, as significantly more patients receiving aripiprazole were dosed within the FDAapproved therapeutic range than the other two cohorts. This discrepancy in dosing within therapeutic range for atypical antipsychotics has been noted in other studies ${ }^{16,25}$ and while it is not clear what the total impact of this is on the results of the current study, it is reasonable to speculate that patients dosed within the appropriate range may be more likely to experience better outcomes.

It is also possible that differential pharmacokinetic properties of the atypical antipsychotics may have impacted the outcomes. Broder et al evaluated hospitalization rates and adherence among patients treated with atypical antipsychotics based on agents with short half-lives compared to agents with longer half-lives. ${ }^{26}$ Agents with longer half-lives (risperidone, olanzapine, and aripiprazole) were less likely to incur a hospitalization or ER visit for mental disorders for a given level of adherence than patients taking an agent with a shorter half-life (quetiapine or ziprasidone). ${ }^{26}$ Since aripiprazole has a long half-life (approximately 75 hours) compared to both olanzapine (37 hours) and quetiapine (6 hours), it may help explain the lower total medical costs and utilization for the aripiprazole patients in the current study, given the impact of half-life on effectiveness, as seen in the Broder et al study. ${ }^{21-23,26}$

\section{Limitations}

As this was a retrospective database study, there was no randomization. Baseline characteristics varied across the cohorts and may have impacted the results. While patients in the aripiprazole cohort had significantly lower PDG scores than patients receiving quetiapine, and significantly lower CCI scores compared to olanzapine and quetiapine, aripiprazole patients had a higher MGH-AD score. Additionally, the aripiprazole cohort had a higher proportion of patients on a preindex mood stabilizer or stimulant, and were more likely to visit a psychiatrist and have a diagnosis of MDD based on an ICD-9 code of $296.2 x$ or $296.3 x$ relative to the other cohorts. By 6 months and with a continuing trend at 12 months, a significantly higher proportion of patients were receiving a dose within the therapeutic range. A multivariate approach was taken in an effort to minimize the bias associated with nonrandomization. Given the nature of claims-data research, information on baseline clinical severity of the patients was not readily ascertainable; however, proxies for disease severity based on available data were created and used in a multivariate framework to control for mitigating circumstances surrounding MDD and other comorbidities. This study considered only total medical costs, and did not incorporate pharmacy (ie, medication) costs into the analysis. Current relative pharmacy costs likely differ from pharmacy costs when this study was conducted due to the recent introduction of generic olanzapine and quetiapine; therefore, for this analysis we focused on the relative total medical costs of patients using each medication, which will likely remain consistent between the study period and the present day. Lastly, the main analysis was conducted on an intent-to-treat basis. This methodology has the potential to introduce some bias, since patients could have discontinued or switched atypical antipsychotic therapy during the study period. However, sensitivity analysis showed that the trends from the main analysis were also present at 6 months.

\section{Conclusions}

Olanzapine and quetiapine were associated with a significantly higher risk of hospitalization and ER visits compared to aripiprazole in commercially insured patients with depression. Furthermore, olanzapine and quetiapine were associated with significantly higher total medical costs. The higher costs may be attributed to higher inpatient costs, supported by the observed higher hospitalization and ER-visit rates. The results of this study demonstrate real-world medical costs and utilization that occurred over a 12-month period following initiation of atypical antipsychotic treatment, and as such may help inform health-care decision-makers in selecting appropriate atypical antipsychotic therapy in patients with MDD. 


\section{Acknowledgment}

Meg Franklin of Franklin Pharmaceutical Consulting assisted with the writing of this manuscript.

\section{Disclosure}

Anagha Nadkarni, Iftekhar Kalsekar, Min You, and Tony Hebden are employees of Bristol-Myers Squibb and hold stock in the company. At the time this manuscript was written, Robert Forbes was employed by Otsuka Pharmaceutical Development and Commercialization.

\section{References}

1. Kessler RC, Berglund P, Demler O, et al. The epidemiology of major depressive disorder. Results from the National Comorbidity Survey Replication (NCS-R). JAMA. 2003;289:3095-3105.

2. Murray CJ, Lopez AD. Evidence-based health policy - lessons from the Global Burden of Disease Study. Science. 1996;274:740-743.

3. Greenberg PE, Kessler RC, Birnbaum HG, et al. The economic burden of depression in the United States: how did it change between 1990 and 2000? J Clin Psychiatry. 2003;64:1465-1475.

4. Ivanova JI, Birnbaum HG, Kidolezi Y, Subramanian G, Khan SA, Stensland MD. Direct and indirect costs of employees with treatmentresistant and non-treatment resistant major depressive disorder. Curr Med Res Opin. 2010;26:2475-2484.

5. Rush AJ, Trivedi MH, Wisniewski SR, et al. Acute and longer-term outcomes in depressed outpatients requiring one or several treatment steps: a STAR*D report. Am J Psychiatry. 2006;163:1905-1917.

6. Connolly KR, Thase ME. If at first you don't succeed: a review of the evidence for antidepressant augmentation, combination, and switching strategies. Drugs. 2011;71:43-64.

7. Fava M. Diagnosis and definition of treatment-resistant depression. Biol Psychiatry. 2003;53:649-659.

8. Crown WH, Finkelstein S, Berndt ER, et al. The impact of treatment-resistant depression on health care utilization and costs. J Clin Psychiatry. 2002;63:963-971.

9. Greenberg P, Corey-Lisle PK, Birnbaum H, Marynchenko M, Claxton A. Economic implications of treatment-resistant depression among employees. Pharmacoeconomics. 2004;22:363-373.

10. Corey-Lisle PK, Birnbaum HG, Greenberg PE, Marynchenko MB, Claxton AJ. Identification of a claims data "signature" and economic consequences for treatment-resistant depression. J Clin Psychiatry. 2002;63:717-726.

11. Russell JM, Hawkina K, Ozminkowski RH, et al. The cost consequences of treatment-resistant depression. J Clin Psychiatry. 2004;65:341-347.
12. Gibson TB, Jing Y, Smith Carls G, et al. Cost burden of treatment resistance in patients with depression. Am J Manag Care. 2010;16: 370-377.

13. American Psychiatric Association. Practice Guideline for the Treatment of Patients with Major Depressive Disorder. 3rd ed. Arlington: American Psychiatric Publishing; 2010.

14. Nemeroff CB. Use of atypical antipsychotics in refractory depression and anxiety. J Clin Psychiatry. 2005;66 Suppl 8:13-21.

15. Papakostas GE, Shelton RC. Use of atypical antipsychotics for treatment-resistant major depressive disorder. Curr Psychiatry Rep. 2008;10:481-486.

16. Jing Y, Kalsekar I, Curkendall SM, et al. Intent-to-treat analysis of health care expenditures of patients treated with atypical antipsychotics as adjunctive therapy in depression. Clin Ther. 2011;33:1246-1257.

17. IMS Health. IMS RxBenchmark: PharMetrics Integrated Database. Available from: http://www.imshealth.com/portal/site/ims/menuitem. d248e29c86589c9c30e81c033208c22a/?vgnextoid=d6952a2e232643 10VgnVCM100000ed152ca2RCRD\&vgnextchannel=c03c90916e442 310VgnVCM100000ed152ca2RCRD. Accessed September 20, 2012.

18. Deyo RA, Cherkin DC, Ciol MA. Adapting a clinical comorbidity index for use with ICD-9-CM administrative databases. J Clin Epidemiol. 1992;45:613-619.

19. Ashcraft ML, Fries BE, Nerenz DR, et al. A psychiatric patient classification system. An alternative to diagnosis-related groups. Med Care. 1989;27:543-557.

20. Petersen T, Papakostas GE, Posternak MA, et al. Empirical testing of two models for staging antidepressant treatment resistance. J Clin Psychopharmacol. 2005;25:336-341.

21. Bristol-Myers Squibb. Abilify (aripiprazole) [package insert]. Princeton: Bristol-Myers Squibb; 2011.

22. Eli Lilly. Zyprexa (olanzapine) [package insert]. Indianapolis: Eli Lilly; 2011.

23. AstraZeneca. Seroquel (quetiapine) [package insert]. Wilmington: AstraZeneca; 2011.

24. Taneja C, Papakostas GI, Jing Y, Baker RA, Forbes RA, Oster G. Cost-effectiveness of adjunctive therapy with atypical antipsychotics for acute treatment of major depressive disorder. Ann Pharmacother. 2012;46:642-649.

25. Leslie DL, Mohamed S, Rosenheck RA. Off-label use of antipsychotic medications in the Department of Veterans Affairs health care system. Psychiatr Serv. 2009;60:1175-1181.

26. Broder MS, Bates JA, Jing Y, Hebden T, Forbes RA, Chang E. Association between second-generation antipsychotic medication half-life and hospitalization in the community treatment of adult schizophrenia. J Med Econ. 2012;15:105-111.
ClinicoEconomics and Outcomes Research

\section{Publish your work in this journal}

ClinicoEconomics \& Outcomes Research is an international, peerreviewed open-access journal focusing on Health Technology Assessment, Pharmacoeconomics and Outcomes Research in the areas of diagnosis, medical devices, and clinical, surgical and pharmacological intervention. The economic impact of health policy and health systems

\section{Dovepress}

organization also constitute important areas of coverage. The manuscript management system is completely online and includes a very quick and fair peer-review system, which is all easy to use. Visit http://www.dovepress.com/testimonials.php to read real quotes from published authors. 